\title{
Optimización simultánea de variables operativas y de diseño de una celda de iones de litio para procesos simples de carga y descarga
}

\section{Simultaneous optimization of operational and design variables of a lithium-ion cell for simple charging and discharging processes}

Presentación: 6-7/10/2020

\section{Doctorando:}

\section{Corina Aimo}

Instituto de Desarrollo y Diseño (INGAR) Consejo Nacional de Investigaciones Científicas y Técnicas (CONICET), Facultad Regional Santa Fe, Universidad Tecnológica Nacional - Argentina

corinaaimo@santafe-conicet.gob.ar

\section{Director:}

\section{Pio Aguirre}

\section{Resumen}

En este trabajo se resumen los principales resultados obtenidos de la aplicación de un modelo fenomenológico de celda de iones de litio en diferentes esquemas de optimización mediante un enfoque de programación matemática. Durante los procesos de carga y descarga de una batería de iones de litio se dan diferentes fenómenos, además, mientras que el proceso de carga se puede predefinir y controlar, los procesos de descarga están sujetos al perfil demandado por determinada aplicación. Debido a esto, se distinguen los esquemas de optimización para cada uno de estos procesos, y a su vez, cada esquema (o problema de optimización) se diferencia principalmente por la función objetivo. Para el proceso de descarga se propone maximizar la energía específica total para un conjunto de valores de tiempo de descarga dados con el objetivo de relacionar energía y potencia entregadas. Para el proceso de carga se consideran tres objetivos distintos basados en los requerimientos de aplicaciones reales; estos son: minimización del tiempo de carga, maximización de la capacidad de carga y minimización de la energía consumida. En ambos esquemas generales (carga y descarga) se plantea la optimización del diseño de celda en diferentes condiciones operativas. Por lo tanto, el enfoque propuesto permite la optimización simultánea de múltiples variables y se obtienen mejoras considerables no sólo en los objetivos evaluados sino también en el desempeño global del sistema.

Palabras clave: Baterías de Litio, Programación Matemática, Restricciones Operativas, Optimización Simultánea del Diseño.

\footnotetext{
Abstract

This work summarizes the main results obtained from the application of a lithium-ion cell phenomenological model in different optimization frameworks using a mathematical programming approach. During the charging and discharging processes of a lithium-ion battery different phenomena occur, in addition, while the charging process can be predefined and controlled, the discharge processes are subject to certain power profile demanded. Due to this, the optimization framework for each of these processes are distinguished, and in turn, each framework (or optimization problem) differs mainly by the objective function. For the discharge process, the objective is the maximization of total specific energy delivered for a set of given discharge time values with the aim of relating delivered energy to delivered power. For the
} 
charging process, three different objectives are considered based on the requirements of real applications; these are: minimization of charging time, maximization of charging capacity and minimization of consumed energy. In both general frameworks (charging and discharging) the cell design optimization under different operating conditions is presented. Therefore, the proposed approach allows the simultaneous optimization of multiple variables and considerable improvements are obtained not only in the evaluated objectives but also in the overall system performance.

Keywords: Lithium Batteries, Mathematical Programming, Operational Restrictions, Simultaneous Design Optimization.

\section{Introducción}

En los últimos años la problemática asociada a la generación, transporte y uso de la energía ha recobrado especial interés. La demanda de energética se encuentra en continuo aumento debido al aumento de las poblaciones, la consecuente expansión urbana y al desarrollo industrial. La generación de energía centralizada de gran tamaño utiliza fuentes convencionales como el carbón, el petróleo y el gas que son limitadas y producen un grave impacto ambiental. La clave para la solución a estos problemas se encuentra en generar procesos sostenibles en el tiempo y al reemplazo de los combustibles fósiles mediante el desarrollo de aplicaciones correspondientes a equipos y procesos altamente integrados para la generación de energía a partir de materias primas renovables, para lograr descentralización e independencia energética.

Se ha demostrado que las celdas de combustible trabajando en conjunto con sistemas de baterías son eficientes para proporcionar una propulsión de cero emisiones en vehículos eléctricos híbridos (HEV) (Yue, Jemei, Gouriveau y Zerhouni, 2019). En un HEV de batería/pila de combustible, el combustible de hidrógeno se convierte en electricidad a través del sistema de pila de combustible a bordo y proporciona la mayoría de las necesidades de energía del vehículo, mientras que las baterías a bordo se utilizan para almacenar energía regenerada y proporcionar la máxima demanda de energía. Esto se debe a que la celda de combustible puede funcionar mejor en condiciones estables y puede alcanzar su máxima eficiencia con carga parcial, mientras que la batería puede funcionar a elevada corriente para compensar los puntos débiles de la celda de combustible. A diferencia de los vehículos eléctricos puros (vehículos eléctricos que solo usan baterías), los HEV de las celdas de combustible del sistema de baterías podrían reducirse en tamaño y, como resultado, disminuiría el peso y el costo general del vehículo.

Para extraer todo el potencial de los sistemas basados en fuentes de energía renovables, específicamente bioetanol e hidrógeno, y maximizar su rentabilidad, las investigaciones deben apuntar a un enfoque holístico a nivel de sistema. El primer paso para alcanzar este objetivo es el modelado de los componentes individuales, considerando una selección adecuada de los subcomponentes de almacenamiento. En este contexto, en el trabajo de tesis que se titula "Modelado, síntesis y optimización de sistemas basados en celdas de combustible con almacenamiento de hidrógeno y de energía", se considerará como componentes de almacenamiento de energía a los sistemas de celdas de combustible de membrana de intercambio protónico (PEMFC) y baterías de litio, debido a su elevada densidad energética y eficiencia cuando trabajan en conjunto, entre otras ventajas. Se han desarrollado modelos matemáticos apropiados que presentan un compromiso entre precisión y complejidad y se utilizaron para el estudio y la optimización de las condiciones operativas y el diseño de estos sistemas de almacenamiento de energía. Luego, se abordará la integración de estos sistemas para satisfacer un perfil potencia demandada que la celda no puede satisfacer debido a su dificultad inherente de cambio de régimen y a los fenómenos de degradación que se producen en la misma. Por ello, las baterías de litio serán las encargadas de absorber los picos y valles de demanda. En este trabajo se resumen los principales resultados obtenidos de la aplicación de un modelo fenomenológico de celda de iones de litio en diferentes esquemas de optimización mediante un enfoque de programación matemática. 


\section{Desarrollo}

En primer lugar, se considera la optimización de las condiciones operativas para un diseño de celda dado. Luego, se plantea la optimización simultánea del diseño de celda (dimensionamiento) junto con las condiciones de operación. Para alcanzar estas metas se utiliza un modelo matemático de celda de litio que predice los principales fenómenos fisicoquímicos de estos dispositivos. El modelo se desarrolló a partir de un enfoque de discretización mediante ecuaciones integrales. Por un lado, se discretizó el dominio radial de partículas esféricas de material sólido activo, obteniendo dos dominios de igual volumen. Por otro lado, el dominio de la longitud de la celda se discretizó, definiendo así los compartimentos electrolíticos de cátodo, separador y ánodo. De esta forma, se obtuvo un modelo de orden reducido que consta de un conjunto de ecuaciones algebraicas y ecuaciones diferenciales ordinarias dependientes del tiempo. Los detalles del desarrollo del modelo y su validación con datos experimentales se pueden encontrar en (Henquín y Aguirre, 2015).

Las condiciones operativas que se estudian y optimizan para el proceso de descarga son: corriente constante (CC), potencia constante (CP) y resistencia constante (CR). Estas condiciones operativas se definen como restricciones explícitas que relacionan a las variables del modelo. La función objetivo de este esquema es maximizar la energía específica total entregada para un conjunto de valores de tiempo de descarga dados con el objetivo de relacionar energía y potencia entregadas.

En el esquema general del proceso de carga las condiciones operativas que se optimizan, que en este caso se denominan protocolos de carga, son las anteriormente nombradas para el caso del proceso de descarga, es decir, CC, CP y CR. Además se estudian protocolos de carga híbridos que consisten en una combinación de dos condiciones operativas distintas durante un mismo proceso de carga, específicamente se estudia el protocolo corriente constante - voltaje constante (CC$\mathrm{CV}$ ), potencia constante - voltaje constante (CP-CV) y el protocolo resistencia constante - voltaje constante (CR-CV). Estos protocolos se definen mediante la imposición de una cota superior en la corriente y la potencia y una cota inferior en la resistencia, respectivamente. Por ejemplo, durante un proceso de carga mediante el protocolo híbrido CC-CV el sistema funciona al valor máximo de corriente durante un cierto tiempo y luego, al valor máximo de voltaje durante el tiempo de carga restante. La proporción del tiempo de carga total en el que funciona la celda en cada una de estas fases (CC o CV) depende del valor predefinido de la cota superior. En este esquema se proponen tres objetivos: P1) minimización del tiempo de carga, P2) minimización de la energía consumida y P3) maximización de la capacidad de carga. Cada uno de estos problemas se diferencia principalmente por la función objetivo (F0). P1 es el problema de carga clásico, en el que el principal desafío es encontrar un equilibrio entre un tiempo de carga corto y una capacidad de carga alta. P2 es equivalente al caso resuelto para el proceso de descarga. En este caso, se dispone de un tiempo relativamente largo y el objetivo es la minimización de la energía consumida, por lo que el protocolo de carga obtenido prioriza la reducción de las pérdidas internas de la celda, lo que podría estar relacionado con una reducción del envejecimiento de celda a largo plazo. Finalmente, el tercer problema (P3) busca reflejar aquellas situaciones en las que se quiere alcanzar la carga máxima en un tiempo muy corto, como en el caso de un vehículo eléctrico. Todos estos problemas de optimización están sujetos a restricciones de seguridad que definen una región factible, que ofrece protección contra los principales fenómenos de degradación de celda que se producen durante el proceso de carga, específicamente para evitar la deposición de litio metálico en el ánodo y la degradación del electrolito en el cátodo.

Además, a partir del Cálculo Variacional fue posible derivar expresiones teóricas de operación óptima tanto para el proceso de carga como para el proceso de descarga. Estas expresiones óptimas relacionan el voltaje de equilibrio, la corriente aplicada y el tiempo de descarga/carga según corresponda. El desarrollo para la obtención de estas expresiones y todos los detalles de los esquemas anteriormente nombrados se encuentra en (Aimo, Henquín, \& Aguirre, 2019)(Aimo y Aguirre, 2020).

Se plantean los siguientes sub-problemas de optimización del diseño para comparar las mejoras relativas logradas con cada una de las principales variables de diseño a nivel de celda: I) Optimización simultánea de espesores de electrodos $\left(L^{C}\right.$ y $\left.L^{A}\right)$, para valores fijos de fracciones volumétricas de porosidad $\left(\varepsilon_{\mathrm{P}}\right)$, sólidos totales $\left(\varepsilon_{\mathrm{S}}\right)$ y masa de celda específica ( $\mathrm{M}_{\text {cell }}$. II) Optimización simultánea de espesores de electrodos y fracciones volumétricas, con masa de celda específica fija en su valor inicial. III) Optimización simultánea de espesores, fracciones de volumen y masa de celda específica. En este caso, la concentración inicial de sal en el electrolito $\left(\mathrm{C}_{\mathrm{e}}{ }^{0}\right)$ también se ha considerado como una variable de optimización adicional.

El modelo fue desarrollado y resuelto en General Algebraic Modelling System (GAMS). Las ecuaciones diferenciales se aproximaron aplicando fórmulas de backward Euler; y así, se obtuvo un conjunto de ecuaciones algebraicas. En todos los 
casos se consideró el tamaño de la cuadrícula de 2000 nodos temporales. El modelo incluye un número total de 96057 ecuaciones algebraicas y 96088 variables. El solucionador CONOP, basado en el método de gradiente reducido, se utilizó para resolver los problemas de optimización.

\section{Resultados}

En la Tabla 1 se presentan los resultados de los diseños óptimos obtenidos para una descarga de 6 horas. Se puede observar la mejora de la función objetivo a medida que aumentan los grados de libertad del problema, es decir, las variables libres en la optimización. Los diseños óptimos DIII se pueden considerar independientes del diseño inicial ya que todas las variables de diseño son optimizadas. En estos casos, se produce un aumento de la masa y de los espesores de los electrodos obteniendo así, celdas de mayor tamaño. Esto es así debido a que la celda inicial considerada puede entregar la máxima energía para descargas de una hora aproximadamente. En relación a las fracciones volumétricas, la tendencia es una reducción de la porosidad (líquido y polímero) y un aumento de sólidos totales en todos los casos, tal que en algunos casos se alcanza la cota superior impuesta de 0,55.

\begin{tabular}{|c|c|c|c|c|c|c|c|c|c|}
\hline $\begin{array}{l}\text { Esquema de } \\
\text { optimización }\end{array}$ & $\begin{array}{l}\text { FO: Eesp } \\
\text { (Wh/kg) }\end{array}$ & $\begin{array}{c}\text { \% } \Delta \text { FO del } \\
\text { diseño } \\
\text { óptimo } \\
\text { contra Do }\end{array}$ & $M_{\text {cell }}\left(\mathrm{g} \mathrm{cm}^{-2}\right)$ & $\mathrm{L}^{\mathrm{C}}(\mathrm{cm})$ & $\mathrm{L}^{\mathrm{A}}(\mathrm{cm})$ & $\varepsilon_{s}^{C}$ & $\varepsilon_{P}^{C}$ & $\varepsilon_{s}^{A}$ & $\varepsilon_{\mathrm{P}}{ }^{A}$ \\
\hline PD-CR-D0 & 97,3 & - & 0,1048 & 0,0190 & 0,0128 & 0,3700 & 0,6300 & 0,4970 & 0,5030 \\
\hline PD-CR-DI & 112,9 & $16,0 \%$ & 0,1048 & 0,0212 & 0,0098 & 0,3700 & 0,6300 & 0,4970 & 0,5030 \\
\hline PD-CR-DII & 135,7 & $39,5 \%$ & 0,1048 & 0,0173 & 0,0108 & 0,5500 & 0,4500 & 0,5500 & 0,4500 \\
\hline PD-CC-DIII & 148,9 & $53,0 \%$ & 0,1532 & 0,0314 & 0,0185 & 0,5162 & 0,4838 & 0,5500 & 0,4500 \\
\hline PD-CP-DIII & 145,5 & $49,6 \%$ & 0,1494 & 0,0306 & 0,0177 & 0,5108 & 0,4892 & 0,5500 & 0,4500 \\
\hline PD-CR-DIII & 150,8 & $55,0 \%$ & 0,1555 & 0,0321 & 0,0189 & 0,5157 & 0,4843 & 0,5500 & 0,4500 \\
\hline PD-OTOP-DIII & 151,2 & $55,4 \%$ & 0,1561 & 0,0324 & 0,0189 & 0,5101 & 0,4899 & 0,5500 & 0,4500 \\
\hline
\end{tabular}

Tabla 1: Valores de la función objetivo y de las variables de diseño alcanzados mediante los esquemas de optimización para diferentes condiciones operativas para una descarga de seis horas. En la segunda columna se calcula la variación porcentual de la energía específica alcanzada por cada diseño óptimo contra el diseño de celda inicial.

Si se comparan los diseños óptimos DIII entre las diferentes condiciones operativas impuestas se puede observar que los diseños son similares para un mismo tiempo de descarga. Se puede concluir que el porcentaje adicional de energía específica está vinculado a una relación entre el voltaje de celda y la corriente aplicada, como lo demuestra la expresión de política de operación óptima teórica (OTOP, obtenida en base al cálculo variacional) y se presenta en la Figura 1, donde se muestran los perfiles de voltaje y corriente de los diseños óptimos obtenidos para la descarga de 6 horas. Los mejores rendimientos después de OTOP se obtienen mediante la operación CR, en ambos casos se puede observar que la corriente acompaña al comportamiento del voltaje, es decir, cuando este último es elevado la celda trabaja a la máxima corriente posible; en cambio, la operación CP presenta el rendimiento más bajo, ya que el comportamiento de la corriente aplicada es inverso al voltaje.

Para el proceso de carga, si se considera el problema de minimización de la energía consumida (P2), que es equivalente al estudiado para la descarga, si bien los mejores resultados son obtenidos con la condición óptima teórica de carga (OTCP), las diferencias con el resto de los protocolos son mínimas, debido a que en este esquema de optimización el tiempo se fija por encima del tiempo mínimo de carga obtenido mediante P1 para un diseño de celda determinado. Es decir, el sistema tiene más tiempo del que necesita para completar la carga, entonces el tipo protocolo de carga que se utilice en esta situación presenta una diferencia marginal. Por otro lado, para los problema P1 y P3, que son equivalentes ya que en ambos casos el sistema siempre buscará trabajar a la máxima corriente posible, los protocolos de carga si generan una diferencia considerable en los valores de la función objetivo, y en estos casos los protocolos de ambos grupos, es decir, híbridos y puros, que presentan el mejor rendimiento corresponden a los casos de CP-CV y CP, respectivamente. Por lo tanto para lograr el mínimo tiempo (o la máxima carga) es conveniente que el proceso de carga comience con una corriente lo más elevada posible. En la Figura 2 se muestran los perfiles de corriente y voltaje en función del mínimo tiempo de carga obtenido para el diseño de celda original (D0). 

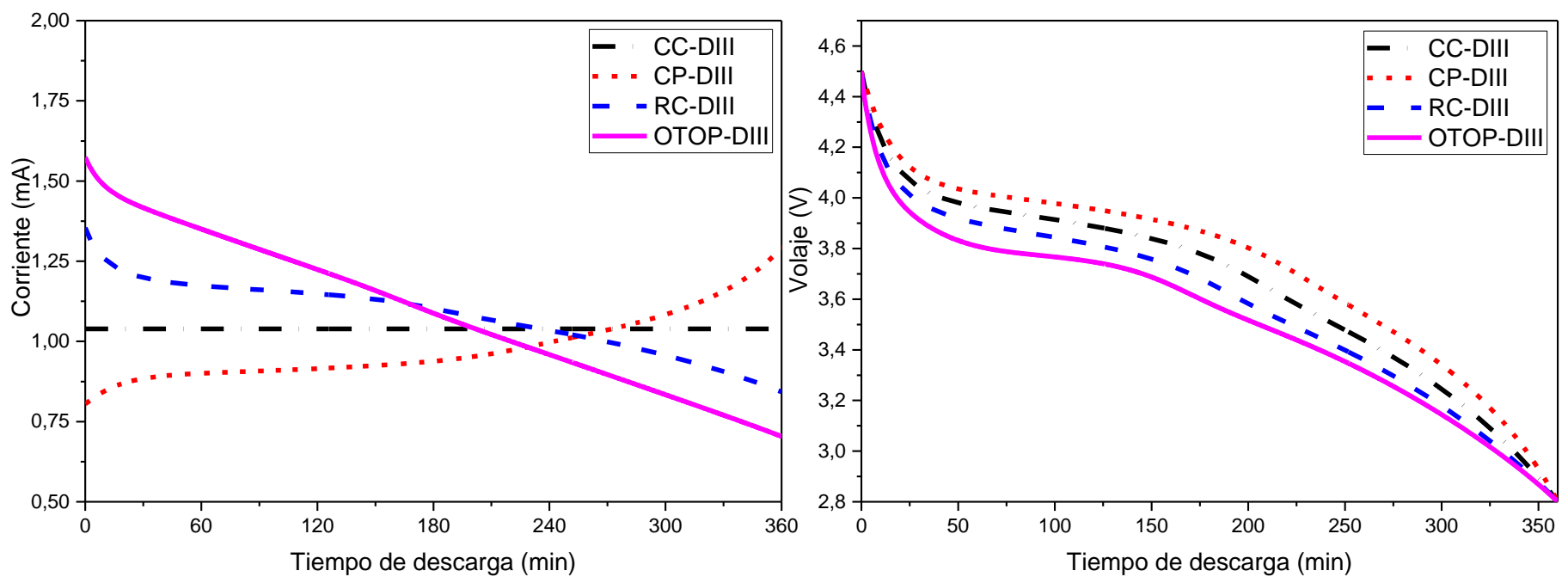

Figura 1: Comparación de los perfiles de corriente y voltaje relación a la condición operativa impuesta, para los diseños óptimos DIII obtenidos para una descarga de 6 horas.
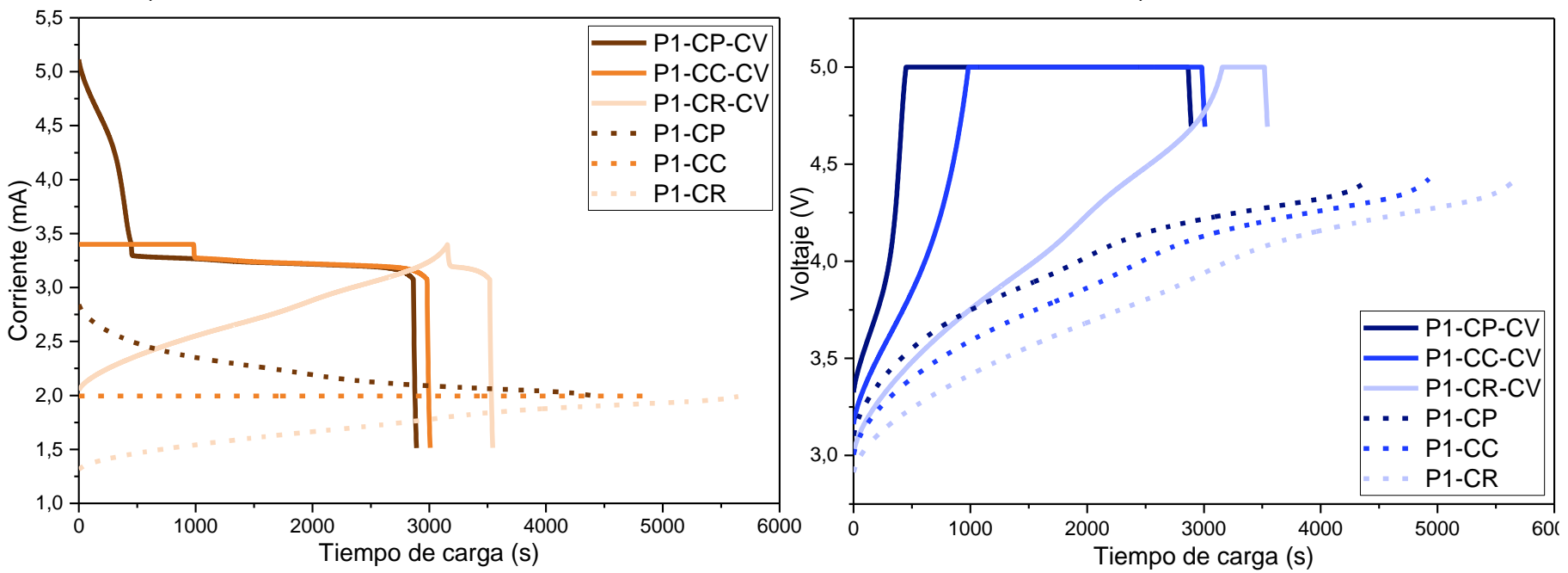

Figura 2: Comparación de los perfiles de corriente y voltaje obtenidos en el esquema de minimización del tiempo de carga en relación a los distintos protocolos de carga impuestos, para el diseño de celda inicial (P1-D0).

En la Tabla 2 se presentan la comparación del diseño óptimo obtenido en la descarga CR al incluir la concentración inicial de la sal en el electrolito como variable de optimización, denotado como $\mathrm{C}_{\mathrm{e}}{ }^{0 \text { opt, }}$, contra diferentes diseños de celda óptimos obtenidos durante el proceso de carga CP. Se consideran los casos de 6 horas de carga y 6 horas de descarga para una comparación equivalente. Se puede observar que los diseños óptimos para la carga poseen valores considerablemente mayores de masa y de espesores de electrodo y se obtiene una reducción de los sólidos con el consecuente aumento de la porosidad en ambos compartimientos, cuando se los compara con el diseño óptimo para el proceso de descarga.

La concentración inicial de la sal en el electrolito se incorporó como variable de optimización debido a que los diseños óptimos con esta variable fija en el valor de $0,001 \mathrm{~mol} \mathrm{~cm}^{-3}$, presentaban como principal fuente de disipación de energía a las caídas de potencial en el electrolito anódico. Con esta variable optimizada (en simultáneo con el resto) se reduce la energía consumida (CE) en un 13\% cuando se compara P2-CP-DIII contra P2-CP-DIII-C $e^{0 o p t}$ y se obtiene un incremento de $38 \%$ de la capacidad de carga (Q) cuando se compara P3-CP-DIII contra P3-CP-DIII-C ${ }^{0}{ }^{0 o p t . ~}$ 


\begin{tabular}{|c|c|c|c|c|c|c|c|c|c|c|}
\hline $\begin{array}{l}\text { Esquema de } \\
\text { optimización }\end{array}$ & $\begin{array}{l}\text { FO: CE } \\
\text { (Wh/kg) }\end{array}$ & $\begin{array}{l}\text { FO: Q } \\
\text { (mAh) }\end{array}$ & $\begin{array}{l}M_{\text {cell }}(g \\
\left.\mathrm{cm}^{-2}\right)\end{array}$ & $\begin{array}{l}\mathrm{L}^{\mathrm{C}} \\
(\mathrm{cm})\end{array}$ & $\begin{array}{l}\mathrm{L}^{A} \\
(\mathrm{~cm})\end{array}$ & $\varepsilon_{s}^{c}$ & $\varepsilon_{P}^{c}$ & $\varepsilon_{s}^{A}$ & $\varepsilon_{\mathrm{P}}{ }^{A}$ & $\begin{array}{l}\mathrm{C}_{\mathrm{e}}{ }^{2} \\
\left(\mathrm{~mol}^{-3}\right. \\
\left.\mathrm{cm}^{-3}\right)\end{array}$ \\
\hline PD-CR-DIII-C $e^{0 o p t}$ & $(155,96)^{*}$ & - & 0,1663 & 0,0337 & 0,0212 & 0,5500 & 0,4500 & 0,5500 & 0,4500 & 0,0019 \\
\hline P2-CP-DIII & 149,38 & $(9,39)^{\star *}$ & 0,2707 & 0,0647 & 0,0510 & 0,3707 & 0,6294 & 0,4000 & 0,6000 & 0,0010 \\
\hline P2-CP-DIII-Ce eopt & 129,48 & $(9,39)^{* *}$ & 0,2864 & 0,0685 & 0,0572 & 0,3500 & 0,6500 & 0,4000 & 0,6000 & 0,0016 \\
\hline P3-CP-DIII & $(158,38)$ & 9,56 & 0,2744 & 0,0655 & 0,0519 & 0,3724 & 0,6276 & 0,4000 & 0,6000 & 0,0010 \\
\hline P3-CP-DIII-Ce ${ }^{0 o p t}$ & $(156,57)$ & 13,17 & 0,3732 & 0,0968 & 0,0715 & 0,3500 & 0,6500 & 0,4000 & 0,6000 & 0,0020 \\
\hline
\end{tabular}

Tabla 2: Valores de la función objetivo y de las variables de diseño alcanzados mediante los esquemas de optimización para una descarga de 6 horas mediante el protocolo CR y para una carga de 6 horas mediante el protocolo puro CP. (*) Para el proceso de descarga la FO es maximizar la energía consumida por unidad de masa, mientras que para el proceso de carga la FO es minimización de la energía consumida. (**) En el esquema de optimización de la carga de P2 estos valores corresponden a una cota inferior.

De todos estos problemas resueltos se observa que a medida que aumentan los grados de libertad del modelo, es decir, las variables que son optimizadas simultáneamente, no solo se logra una mejora en la función objetivo evaluada, sino que también se obtiene una mejora en el desempeño global del sistema, y en el caso de una celda de iones de litio esto se ve reflejado, por ejemplo, en una distribución más equitativa de las pérdidas de energía debidas a las caídas de potencial en las fases líquidas y sólidas y a los sobrepotenciales (Aimo y Aguirre, 2020).

\section{Conclusiones}

Los resultados presentados en este documento corresponden a trabajos relacionados con la optimización de una celda de iones de litio mediante el estudio de diferentes escenarios (esquemas de optimización), con el objetivo de identificar a las variables operativas y de diseño más influyentes en el desempeño de estos dispositivos durante procesos simples de carga y descarga. Un resultado destacable de este análisis es que si bien los diseños óptimos obtenidos son bastante independientes de la condición operativa impuesta, se presentan grandes diferencias cuando se compara un diseño óptimo para la carga contra uno para la descarga. Por lo tanto, con el desarrollo de estos trabajos se deja en evidencia que los procesos de carga y descarga son de diferente naturaleza y se logra una comprensión sistémica de la mejora del rendimiento de una celda de iones de litio para cada proceso. En este sentido, se establecen las bases necesarias para trabajos futuros relacionados con problemas de optimización de múltiples variables para procesos cíclicos (secuencias de carga, descarga, y apagado) de baterías y, además, para la adaptación y aplicación de este estudio a los sistemas de PEMFC incorporando los modelos desarrollados a esquemas de optimización similares, para la posterior integración de estos componentes en un sistema que pueda entregar perfiles de potencia que simulen la demanda de aplicaciones reales.

\section{Referencias}

Aimo, C. E., \& Aguirre, P. A. (2020). Lithium-ion whole-cell design and charging protocol optimization within safe operating conditions. Journal of Energy Storage, 30(May), 101515. https://doi.org/10.1016/j.est.2020.101515

Aimo, C. E., Henquín, E. R., \& Aguirre, P. A. (2019). Optimal Design and Discharge Operation of Lithium-Ion Whole-Cell. Journal of Energy Storage, 24(August), 1-9. https://doi.org/.1037//0033-2909.I26.1.78

Henquín, E. R., \& Aguirre, P. A. (2015). Phenomenological Model-Based Analysis of Lithium Batteries: Discharge, Charge, Relaxation Times Studies, and Cycles Analysis. AiChE Journal, 61(1), 90-102. https://doi.org/10.1002/aic

Yue, M., Jemei, S., Gouriveau, R., \& Zerhouni, N. (2019). Review on health-conscious energy management strategies for fuel cell hybrid electric vehicles: Degradation models and strategies. International Journal of Hydrogen Energy, 44(13), 68446861. https://doi.org/10.1016/j.ijhydene.2019.01.190 